\title{
Electromagnetically Driven Micropump with Magnetic-fluid-sealed Thrust Bearing Contributing to Miniaturization of Medical Analytical Sensor Systems
}

\author{
Fumihito Kato, Naoya Oshida, Yu Sato, Noriyasu Masumoto, and Xiaoyou Zhang \\ Nippon Institute of Technology, Faculty of Fundamental Engineering, Department of Mechanical Engineering \\ 4-1 Gakuendai, Miyashiro-machi, Minamisaitama, Saitama 345-8501, Japan
}

(Received May 20, 2019; accepted September 2, 2019)

Keywords: micropump, magnetic fluid seal, thrust bearing, electromagnetic drive, biosensor

In this study, we developed an electromagnetically driven micropump with a magnetic fluid seal, which greatly contributes to the miniaturization of medical analytical sensor systems. The magnetic fluid seal applied to this micropump prevented the solution from entering the inside of the thrust bearing attached to the gap between the liquid feed rotor and the stator, and the abrasion powder generated from the bearing was prevented from being mixed into the solution. Furthermore, because the liquid feed rotor was rotated in a noncontact manner by the electromagnetic force from the coil attached to the external pump, continuous feeding of liquid with reduced pulsation was possible. When the rotational speed of the liquid feed rotor was 500 rotations per minute, the feed rate of pure water was $2 \mathrm{ml} / \mathrm{min}$. At this rotational speed, there was no pure water intrusion into the thrust bearing nor separation of the magnetic fluid from the neodymium magnet. This result indicates that the magnetic fluid seal for the thrust bearing is effective. The micropump developed is a device that can be integrated with a biosensor chip, thereby contributing to the realization of a compact medical analysis sensor system that can be easily operated beside a patient.

\section{Introduction}

Biosensors are attracting attention as a tool for early detection of intractable diseases such as cancer and diabetes and for the development of antibody drugs. Quartz crystal microbalance (QCM) and surface plasmon resonance (SPR) biosensors are capable of measuring biomolecular reactions in real time and quantitatively evaluating affinity. The QCM sensors detect mass and utilize the frequency shift attributed to the mass increase when the target substance is adsorbed on the surface of a thin quartz oscillator excited by thickness shear vibration. ${ }^{(1-4)}$ The

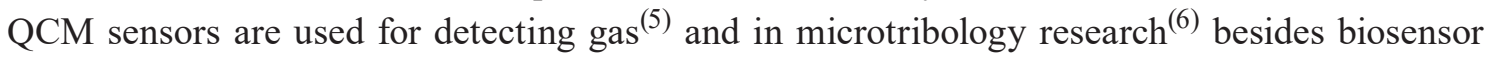
applications. The SPR sensors are optical sensors that utilize the resonance of evanescent and surface plasmon waves. By causing the light to enter a prism on which a metal thin film is formed so as to totally reflect the light, an evanescent wave shorter than the wavelength of the

*Corresponding author: e-mail: kato.fumihito@nit.ac.jp

https://doi.org/10.18494/SAM.2019.2437 
light is generated on the surface of the metal thin film. At the same time, a surface plasmon wave is excited. Since the resonance angle of two waves changes when a biomolecular reaction occurs on the surface of the metal thin film, the SPR can be used for biosensing, ${ }^{(7-10)}$ and is widely used in medical analysis sensor systems ${ }^{(11)}$ because it can be easily accommodated into multichannels. ${ }^{(12)}$

A medical analysis sensor system using QCM mechanically clamps a thin quartz oscillator with a dedicated fixture made of metal; therefore, it easily breaks when attaching or removing it. Accordingly, special skill is required in the operation. In addition, because the temperature characteristics change owing to the difference in thermal expansion coefficient between the quartz and the fixture, a high-precision temperature control device is required. As a result, the whole system becomes large. A medical analysis sensor system using SPR induces changes in dielectric constant on the detection area even if the target substance is close to the detection surface. Therefore, the correction of background noise due to permittivity changes is required; accordingly, adjustment for evaluation is extremely difficult. In addition, a high-precision temperature control device is required to reduce changes in the dielectric constant of the buffer solution and the target substance owing to changes in ambient temperature; therefore, the whole system becomes large, as in the system using QCM. As a common issue to the above two medical analysis sensor systems, the connections of several tubes between the sensor element and the liquid feeding pump become complicated when using multiple channels.

In order to resolve these issues, a wireless electrodeless QCM biosensor chip formed by nanoimprint with polydimethylsiloxane (PDMS) was developed, assuming the integration of a liquid-feeding pump and a sensor chip. ${ }^{(13,14)}$ This sensor chip has the unique structure that an AT-cut quartz oscillator having the resonance characteristic of several $\mathrm{ppm} /{ }^{\circ} \mathrm{C}$ at room temperature ${ }^{(15)}$ is supported by micropillars without being mechanically fixed in a microchannel. The sensor is driven by applying an electromagnetic wave from one antenna mounted outside the chip to excite the quartz oscillator through the reverse piezoelectric effect, and at the same time, receiving charges caused by the piezoelectric effect generated on the quartz surfaces by the other antenna. As mentioned above, because the quartz oscillator can freely move in the microchannel, the structural damping at resonance excitation is significantly decreased; moreover, the temperature control system is not required in the wireless driving of the AT-cut quartz oscillator.

Existing micropumps include the diaphragm pump, syringe pump, peristatic pump, and electromagnetic drive pump. The diaphragm pump has the check valve and feeds the solution by moving the diaphragm installed on the pump chamber up and down using a piezoelectric actuator or a solenoid coil. ${ }^{(16,17)}$ Although this pump is small and can provide a large output, it generates pulsations. The syringe pump is composed of an outer cylinder and an inner cylinder and feeds solution by gradually pushing the solution into the inner cylinder. ${ }^{(18,19)}$ This pump can feed a small volume of solution with high accuracy, but not continuously for a long time. The peristatic pump feeds a solution by repeatedly compressing and restoring the tube by rotation of the roller. ${ }^{(20,21)}$ This pump is capable of feeding highly viscous solutions; however, it can generate pulsations and cause wear on rotating rollers and gears, and damage to the tube. The electromagnetically driven pump supplies a solution by noncontact rotation of the liquid 
feed rotor levitated by the electromagnetic force in the pump chamber. ${ }^{(22,23)}$ Although this pump can be used semipermanently without mechanical contact, it requires an external sensor because feedback control is performed to constantly float the rotor, and it is difficult to restrict the shape and miniaturize the pump.

As described above, these various pumps have different drive systems that are already used for existing pumps used in commercial medical analysis sensor system. These existing pumps are difficult to be directly integrated with biosensor chips. In addition, even if they can be integrated with biosensor chips, there are also issues regarding the size of the analysis system and the durability of the parts of the solution-feeding pump. Therefore, at present, the sensor chips and the solution-feeding pump are used independently, and a compact and easy-to-use medical analysis sensor system that can be used beside a patient has not been realized.

In this study, we developed a novel micropump that can miniaturize the medical analysis sensor system, has excellent durability, and can be directly bonded to a PDMS-QCM biosensor chip. This micropump has a unique structure in which the thrust bearing is sealed by a magnetic fluid. Consequently, the wear powder generated from the driving parts of the micropump does not mix with the solution, and the solution does not enter the inside of thrust bearing. Therefore, this micropump is hygienic and excellent in durability. This micropump and a PDMS-QCM biosensor chip can be integrated by direct bonding. Therefore, the tubes connecting between the sensor element and the micropump are not necessary; accordingly, a medical analysis sensor system can be miniaturized. In addition, the operation is also simplified because the tubes do not need to be attached and detached.

As the aim is to develop a micropump that contributes to the miniaturization of medical analysis systems, the dimensions of the pump were decided to be such that the pump can be placed on the palm as one of the numerical target values. Specifically, the height, depth, and width were each decided to be $60 \mathrm{~mm}$ or less. The solution feeding performance was decided to be several microliters per minute, that is, at levels that can be used in medical analysis sensor systems. In addition, it was also considered as a pump performance index that no seal peeling occurred during solution feeding. By integrating the micropump and the PDMS-QCM biosensor chip and miniaturizing the medical analytical sensor system, the burden on the patient can be greatly reduced because a quick examination and diagnosis beside the patient, such as point-of-care testing (POCT), is possible.

\section{Structure of Magnetic-fluid-sealed Electromagnetically Driven Micropump}

The exterior and cross section of the micropump proposed in this study are shown in Fig. 1. The outer dimensions of the pump are a diameter of $50 \mathrm{~mm}$ and a height of $50 \mathrm{~mm}$, and the diameter of liquid feed/waste port is $3 \mathrm{~mm}$. However, the dimensions of the electromagnetic drive coil are not included. This pump is composed of three parts: upper and lower stators and a liquid feed rotor. The upper stator has a truncated cone shape that serves to direct the solution in the pump to the waste port and encloses the liquid feed rotor. The lower stator has a flat structure for installing the coil for the electromagnetic drive. The liquid feed rotor is a monolithic structure of blades for agitating and feeding the solution and a hollow ring for 


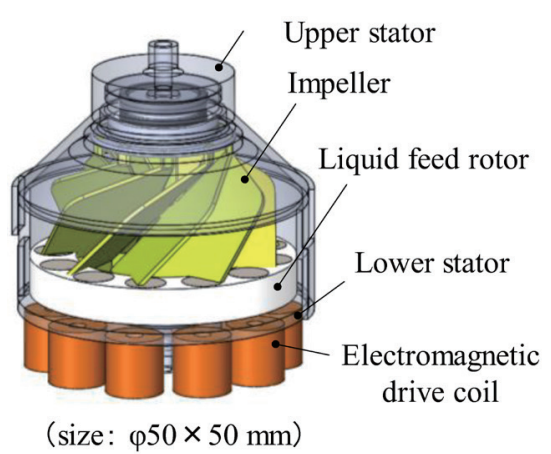

(a)

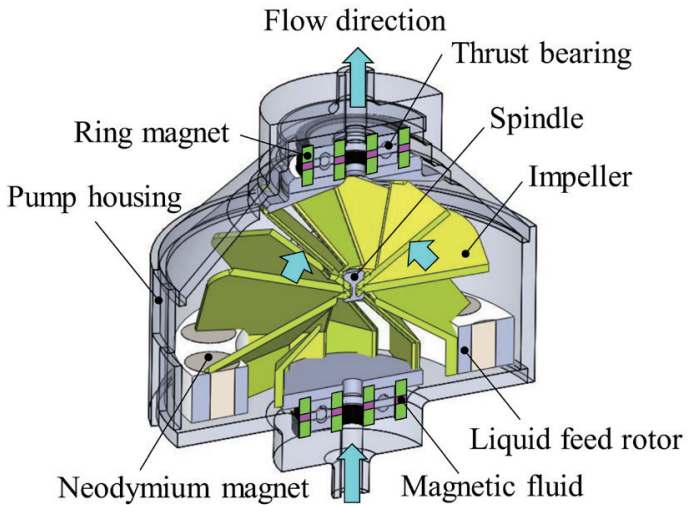

(b)

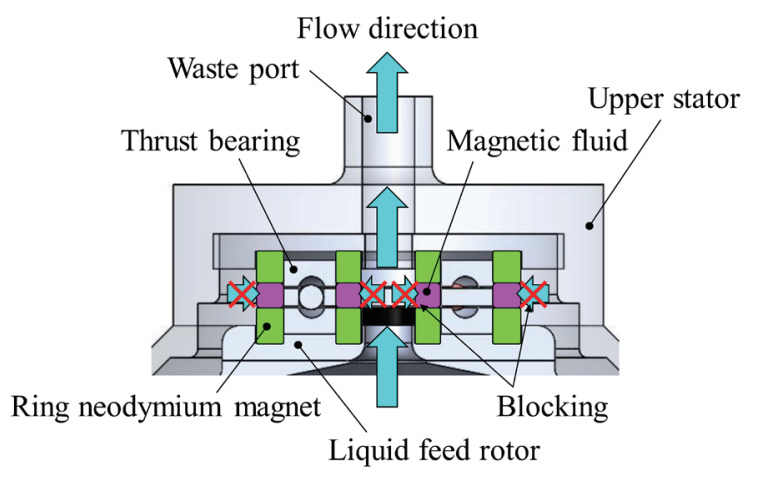

(c)

Fig. 1. (Color online) Electromagnetically driven micropump with magnetic-fluid-sealed thrust bearing: (a) appearance of device, (b) cross section (internal structure), and (c) enlarged view of magnetic-fluid-sealed thrust bearing.

embedding the neodymium magnets. Furthermore, the liquid feed rotor is supported by the upper and lower stators via two magnetic-fluid-sealed thrust bearings. Ten electromagnetic drive coils (diameter, $10 \mathrm{~mm}$; height, $10 \mathrm{~mm}$ ) are installed on the lower stator surface for noncontact driving of the liquid feed rotor by electromagnetic force. These coils have a round metal bar as a core, and the copper wires of adjacent coils are reversely wound. Twelve neodymium magnets (diameter, $7 \mathrm{~mm}$; height, $6.5 \mathrm{~mm}$; magnetic flux density, $460 \mathrm{mT}$ ) are embedded in the hollow ring of the liquid feed rotor. The neodymium magnets are installed such that the polarities of adjacent magnets are opposite to each other. A pair of ring-shaped neodymium magnets are attached to the surfaces of the rotor and stator facing each other, and the fluorine-based magnetic fluid having excellent corrosion resistance is retained between the pair of neodymium magnets, making up the magnetic fluid seal for the thrust bearing. As a result, wear powder generated from the components constituting the bearing can be prevented from being mixed into the solution, and the solution can be prevented from entering the inside of the bearing. As a result, solution contamination, foreign material mixing, and bearing corrosion 
do not occur. In this micropump, because the liquid feed rotor is rotated in a noncontact manner by the attractive and repulsive electromagnetic forces, continuous liquid feeding with reduced pulsation becomes possible. Furthermore, because the mechanical contact and friction are reduced by the noncontact driving, a considerably longer life can be expected for this pump than for the conventional micropump. In addition, because thermoplastic resin [e.g., polymethyl methacrylate (PMMA)] is used for the micropump housing, the housing can be directly bonded to the PDMS substrate by covalent bonding via oxygen atoms. ${ }^{(24,25)}$ Direct bonding does not result in internal stresses due to differences in the thermal expansion coefficient between different materials, and neither the micropump nor the PDMS-QCM biosensor chip warps after direct bonding. As a result, the characteristics of the device are not deteriorated.

\section{Design and Fabrication}

\subsection{Structural design by electromagnetic analysis and flow analysis}

To improve the structural design of the micropump, the shape of the liquid feed rotor was investigated by electromagnetic field analysis and flow analysis. First, the electromagnetic force generated between the neodymium magnets embedded in the liquid feed rotor and the electromagnetic drive coil was calculated, and then the rotor torque was estimated using the calculated result. Because the positional relationship between the neodymium magnet and the electromagnetic drive coil is in the same phase each time it rotates $6^{\circ}$ from the state where the center axes of the coil and the magnet coincide, electromagnetic field analysis was carried out at $0-6^{\circ}$. Generally, as the current applied to the coils increases, the magnetic field strength of the electromagnet increases, but the allowable current density of the copper wire is about $10 \mathrm{~A} / \mathrm{mm}^{2}$. Because an enameled wire with an insulating coating film thickness of $0.01 \mathrm{~mm}$ and a copper wire diameter of $0.28 \mathrm{~mm}$ was wound for 200 turns on the electromagnetic coil drive, the allowable current became $0.62 \mathrm{~A}$ in this micropump. In the electromagnetic field analysis, the rotor torque for the rotor rotation angle was calculated taking into consideration the above conditions. At this time, the influence of the inertial force due to the rotation of the liquid feed rotor is ignored. Figure 2 shows the torque applied on the liquid feed rotor calculated by electromagnetic field analysis. Because the rotor torque becomes positive in all regions, continuous rotation of the rotor can be expected.

\subsection{Structural design by fluid analysis}

The flow velocity distributions in the micropump at a rotational speed of 1000 rotations per minute of the liquid feed rotor were calculated assuming pure water as the liquid feed medium. Then, the relationships among the flow velocity and the pressure at the waste liquid port for the blade angle and the number of blades were investigated.

Figure 3 shows the flow velocity distribution with and without a spindle for bundling liquid feed blades. In a spindleless construction, the solution flows linearly from the liquid feed port to the waste port without agitation in the pump, resulting in stagnation near the side wall of the 


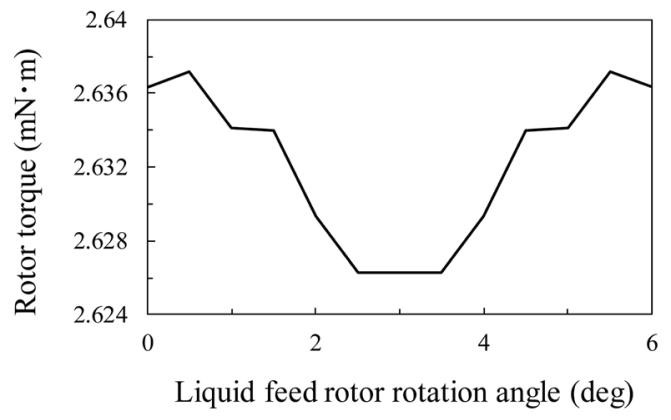

Fig. 2. Relationship of the rotor torque with rotor rotation angle $\left(0-6^{\circ}\right)$.

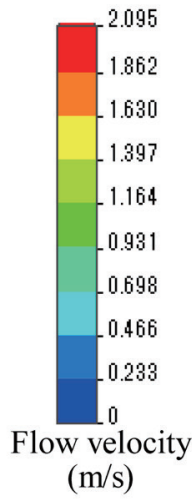

$(\mathrm{m} / \mathrm{s})$

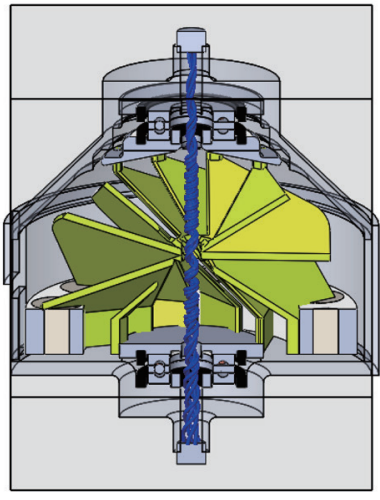

(a)

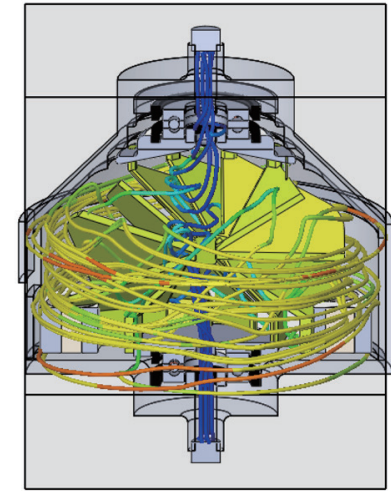

(b)

Fig. 3. (Color online) Flow velocity distribution in the pump: (a) without the spindle and (b) with the spindle.

pump housing. Therefore, when different solutions are sequentially fed, there is a problem that they mix and react in the pump. On the other hand, in the structure with the spindle, because the solution is stirred entirely in the pump, the stagnation of the solution can be reduced. From these results, the structure having a spindle was adopted to the liquid feed rotor of the present micropump.

Next, the relationships of flow velocity and pressure at the waste liquid port with the blade angle were investigated. In this investigation, each blade was assumed to be a flat plate with a thickness of $1 \mathrm{~mm}$, and there were nine blades. The blade angles were also investigated in the range of $40-50^{\circ}$ while considering the pump geometry. The results of the analysis are shown in Fig. 4. At blade angles of $45^{\circ}$ or more, the flow velocity and the discharge pressures are almost flat. From this result, the blade angle of $45^{\circ}$ was selected because there is a wide gap between impellers and is easy to adopt in the manufacture of the pump.

Next, the flow velocity and pressure of the waste liquid port with respect to the number of blades were investigated. The number of blades was 3-12. The results of analysis are shown in Fig. 5. As the number of blades increases, the pressure increases monotonically, but the flow velocity tends to reach a peak and then begins to decrease in the case of 6-9 blades. From 


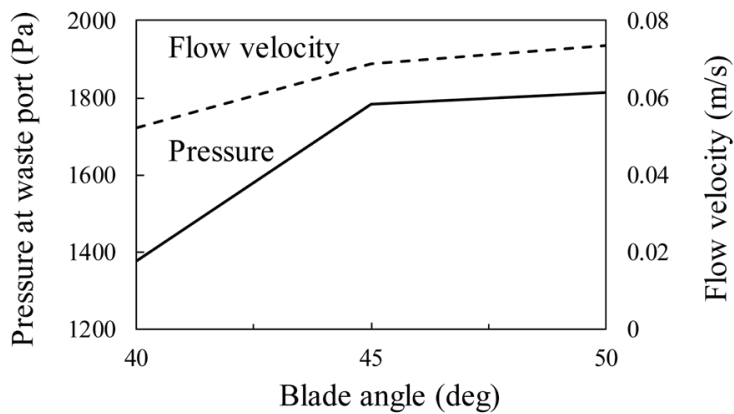

Fig. 4. Relationships of flow velocity and pressure with blade angle.

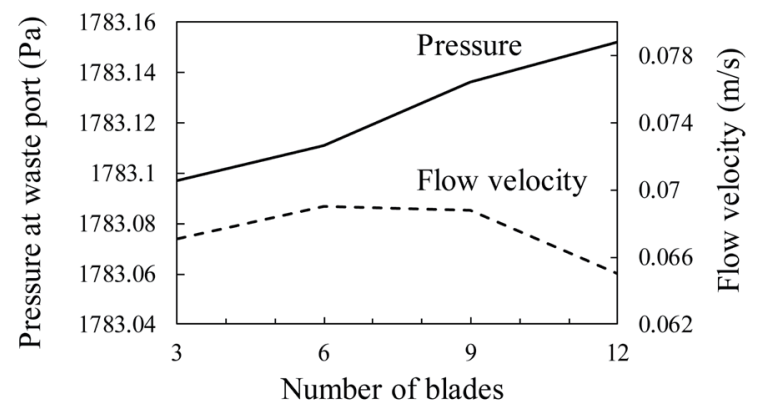

Fig. 5. Relationships of pressure and flow velocity with number of blades.

this result, the number of blades was selected to be nine, at which high values of pressure and flow velocity were obtained. In this analysis, the flow velocity in the waste liquid port with a diameter of $3 \mathrm{~mm}$ is about $30 \mathrm{ml} / \mathrm{min}$.

\subsection{Production of parts using 3D printer}

We designed the parts of the micropump with a manufacturing tolerance of $\pm 0.1 \mathrm{~mm}$ and an assembly tolerance of $\pm 0.3 \mathrm{~mm}$. The machining center is generally applied to manufacture parts that require such high precision dimensional accuracy. However, in this study, the 3D printer was chosen from the perspective of manufacturing time, cost, and precision for parts made of resin. The most commonly used 3D printers employ the fused deposition modeling (FDM) method, in which products are formed by melting and laminating filaments such as those of ABS resin. This shaping method is not suitable for manufacturing thin plate parts such as an impeller. On the other hand, the material jetting (MJ) method can realize highly accurate lamination (e.g., $0.05 \mathrm{~mm}$ ) of resin layers in the height direction. Therefore, it is easy to form thin and complex structures like the impeller with submillimeter tolerance. For these reasons, the MJ method was applied to form the liquid feed rotor and two stators in this study. In the flow of fabrication by the MJ method, photosensitive resin ejected from an ink jet head is cured by ultraviolet radiation. In the fabrication, in addition to the formation of the parts that compose the micropump, the support structure that is to be finally removed is also formed at the same time. In addition, because thermoplastic resin such as PMMA is used as the photosensitive resin, direct bonding with the PDMS-QCM biosensor chip will be possible. Organic solutions and strong acid solutions are often used in the measurement protocols for biomolecular reactions using the biosensor. Therefore, it is necessary to improve the corrosion resistance of the inside of the micropump by coating it with, for example, fluorine-based resin. In this study, we intended to carry out a fundamental analysis of the usefulness of the magneticfluid-sealed thrust bearing for a micropump; therefore, we did not consider corrosion resistance when fabricating the parts. The upper and lower stators and the side wall fabricated by the MJ method were hermetically fixed using an adhesive. The electromagnetic drive coil and the 
lower stator were also similarly fixed using the adhesive. Figure 6(a) shows the appearance of the liquid feed rotor incorporated in the micropump. Figure 6(b) shows the appearance of the electromagnetically driven micropump with the magnetic-fluidsealed thrust bearing after assembly.

\section{Experimental Procedure}

\subsection{Frequency characteristic of electromagnetic drive coil}

The twelve electromagnetic drive coils are arranged in close proximity, leading to parasitic capacitance between the coils. Therefore, it is assumed that transmission loss will occur as the alternating current (AC) applied to the electromagnetic drive coil becomes high in frequency. Accordingly, considering an equivalent circuit of the entire coil and identifying each parameter, the impedance-frequency characteristic was calculated. Next, the actual impedance-frequency characteristic of the electromagnetic drive coil was measured using an impedance analyzer (IM3570, HIOKI). The calculation result obtained using the equivalent circuit and the measurement result obtained using the impedance analyzer were compared to confirm the transmission loss due to the parasitic capacitance of the $\mathrm{AC}(\sim 100 \mathrm{~Hz})$ applied to the electromagnetic drive coil.

\subsection{Torque of liquid feed rotor}

The torque of the liquid feed rotor caused by the attractive and repulsive forces between the electromagnetic drive coil and the neodymium magnet was measured. In the torque measurement, a wire was wound around the liquid feed rotor, and the end of the wire was connected to a force gauge (DS2-5N, Imada) to measure the force in the tangential direction of

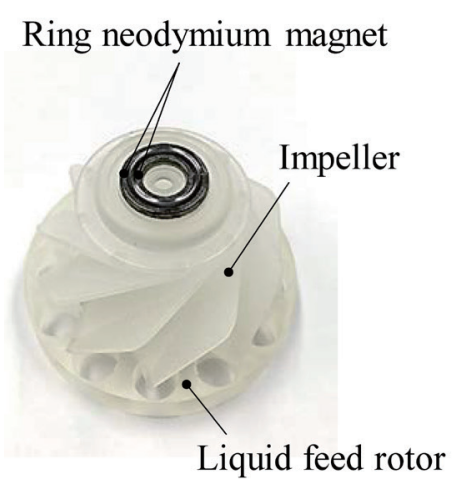

(a)

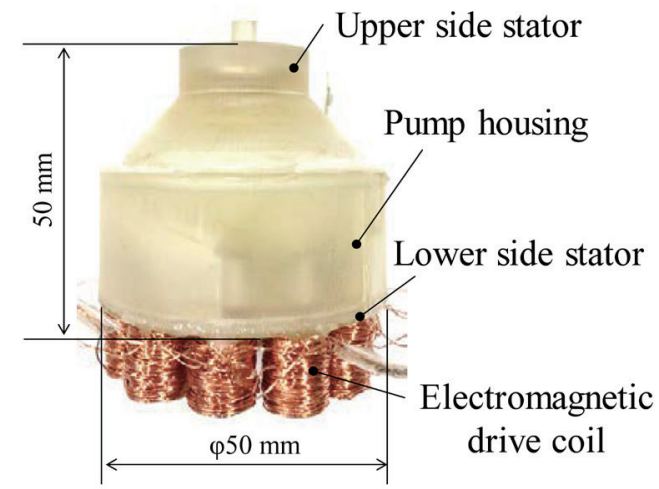

(b)

Fig. 6. (Color online) Appearance of liquid feed rotor and micropump fabricated by MJ method. (a) Liquid feed rotor comprising an impeller with nine feeding blades. (b) External view of electromagnetic drive micropump after assembly. 
liquid feed rotor. The torque relative to the rotor center axis was calculated from the obtained tangential force. The measurements were carried out in air with a direct current (DC) of $0.62 \mathrm{~A}$, and the maximal torques at $0-6^{\circ}$ were measured.

\subsection{Pumping performance of micropump}

To evaluate the liquid feeding performance, the discharge pressure and flow rate with respect to the rotation speed of the liquid feed rotor were measured. The number of revolutions was calculated from the cycle of the output pulse signal using a small neodymium magnet embedded in the ring portion of the liquid feed rotor and a Hall IC attached to the outside of micropump housing. Figure 7 shows the state of the liquid feeding experiment. In this experiment, the liquid feed/waste beakers, the pump interior, and the tube interior were filled with pure water as an initial state. At this time, the water levels in the liquid feed/waste beakers are the same. The liquid feed rotor is rotated in a noncontact manner by applying $\mathrm{AC}$ to the electromagnetic drive coil. The pure water is sucked up from the liquid feed beaker and discharged to the liquid waste beaker. The discharge pressure was calculated from the change in the water level of the liquid waste beaker at this time. The flow rate was calculated from the amount of waste liquid generated per unit time. Furthermore, the performance of the magnetic fluid seal with respect to the rotation speed of liquid feed rotor was evaluated. Specifically, we confirmed whether or not pure water entered into the thrust bearing at an arbitrary rotation speed and whether or not the magnetic fluid seal peeled off. As the gap height for retaining the magnetic fluid is smaller and the area is larger, the magnetic fluid sealing performance of the thrust bearing is improved. However, the clearance height for retaining the magnetic fluid was decided to be $1 \mathrm{~mm}$ considering the runout of the liquid feed rotor rotating at high speed and expansion due to heat generation. This is a sensible height in terms of manufacturing and assembly tolerances. As the clearance height increases, the magnetic fluid is more likely to be peeled by the increase in flow

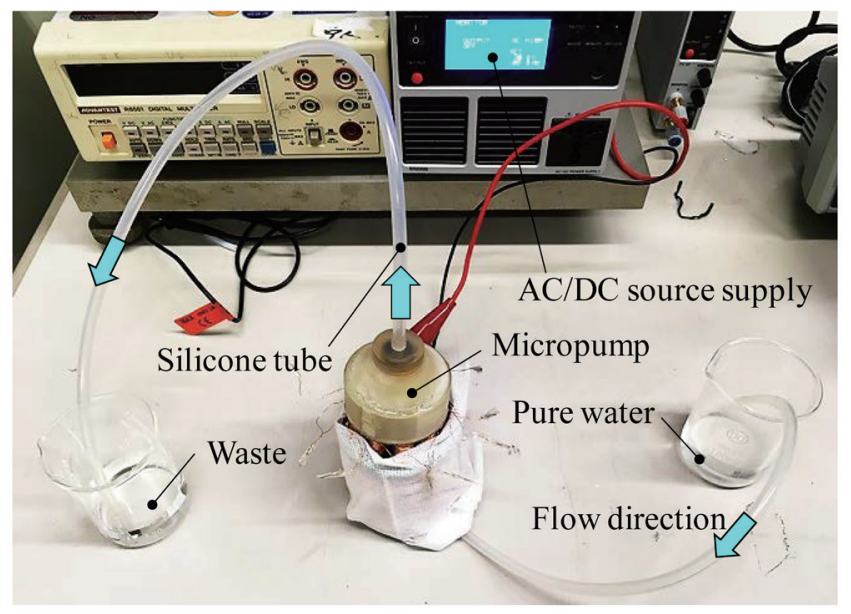

Fig. 7. (Color online) Setup of flow performance experiment of micropump with magnetic-fluid-sealed thrust bearing. 
velocity and pressure depending on the rotational speed of the liquid feed rotor. Therefore, it is necessary to evaluate the separation of the magnetic fluid with respect to the rotational speed of the liquid feed rotor.

\section{Result and Discussion}

\subsection{Results of frequency characteristic evaluation of the electromagnetic drive coil}

The ten electromagnetic drive coils are circularly arranged on the surface of the lower stator. The adjacent electromagnetic drive coils are wound in opposite turns. When the liquid feed rotor is driven by the electromagnetic force, AC is applied to the coil [Fig. 8(a)]; therefore, as the frequency increases, the resonance or dip at a specific frequency may occur owing to the transmission loss due to the parasitic capacitance between the coils. Accordingly, the impedance characteristic of the electromagnetic drive coil was investigated. As shown in Fig. 8(b), the electromagnetic drive coil was regarded as an equivalent circuit in which the inductor $L$ and the resistor $R$ were connected in series and the parasitic capacitance $C_{p}$ was connected in parallel. At this time, assuming that the angular frequency of AC to be $\omega$, the impedance $\dot{Z}$ can be expressed by

$$
\dot{Z}=\frac{R^{2}+\omega^{2} L^{2}}{R+j \omega\left(R^{2} C_{p}+\omega^{2} C_{p} L^{2}-L\right)} .
$$

The values of $L, C_{p}$, and $R$ constituting the equivalent circuits were obtained. Using the impedance analyzer and $0.43 \mathrm{mH}, 3.99 \mathrm{pF}$, and $17.1 \Omega$, the impedance characteristic of the equivalent circuit was calculated with Eq. (1) (theoretical value). Subsequently, the electromagnetic drive coil was connected to the impedance analyzer, and then the impedance

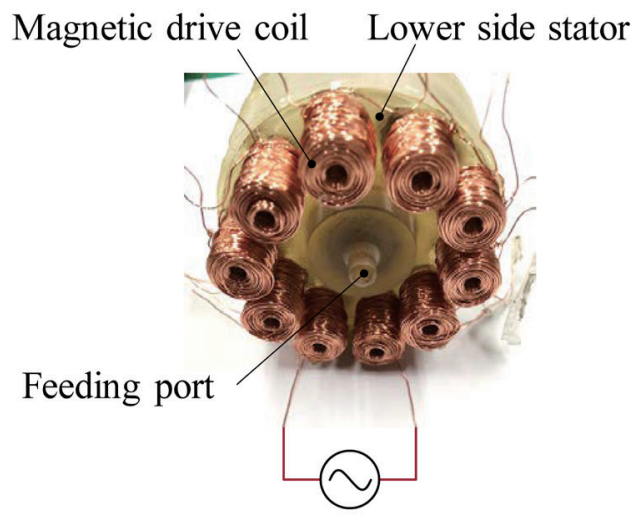

(a)

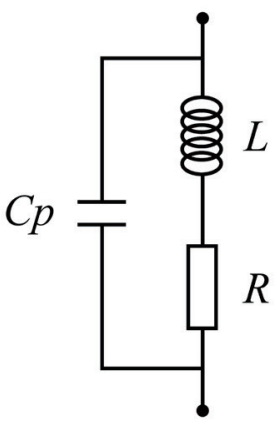

(b)

Fig. 8. (Color online) (a) Electromagnetic drive coil fixed to the lower stator. (b) Equivalent circuit considering parasitic capacitance. 
characteristic was measured (measured value). Figure 9 shows the respective results in the frequency band $10-1000 \mathrm{~Hz}$. The theoretical value is steady up to $100 \mathrm{~Hz}$, but slightly increases linearly above $200 \mathrm{~Hz}$. On the other hand, the measured values become steady up to $100 \mathrm{~Hz}$, and then increase exponentially above $200 \mathrm{~Hz}$. The theoretical and measured results are steady up to $100 \mathrm{~Hz}$ and can be used without current transfer losses at particular frequencies because there are no resonances or dips due to parasitic capacitance. Above $200 \mathrm{~Hz}$, the measured value increases exponentially with respect to the theoretical value, which increases almost linearly, and therefore, it is found that current transfer loss not caused by parasitic capacitance occurs. Because the $\mathrm{AC}$ applied to the electromagnetic drive coil is in the low-frequency band (up to $110 \mathrm{~Hz}$ ), the coil can be used stably without causing any current transmission loss.

\subsection{Results of torque measurement of liquid feed rotor}

If the torque of the liquid feed rotor is not sufficient, the liquid feed rotor cannot be rotated because of the viscosity resistance of pure water. Therefore, the force gauge was used to measure the tangential force acting on the liquid feed rotor, and then the rotor torque was calculated. At this time, a DC of 0.62 A was applied to the electromagnetic drive coil, and it was evaluated in the range of $0-6^{\circ}$ in air. Figure 10 shows the relationship of the rotor torque with the rotation angle of the liquid feed rotor. The experimental result was reduced by about $54 \%$ at maximum compared with the result of the electromagnetic field analysis. Two main factors are considered for this difference. The first is the effect of heat loss due to the current. Because a current having the maximum allowable current density is applied to the electromagnetic drive coil, it is assumed that the electromagnetic force is deteriorated by the heat loss due to the heat generated from the coil, and as a result, torque is reduced. The second is the effect of friction loss due to an increase in the load that the thrust bearing receives. In addition to the attractive force between the neodymium magnets of the magnetic fluid seal attached to the liquid feed rotor and the upper and lower stators, it is presumed that the force by which the electromagnetic drive coil attracts the neodymium magnets embedded in the

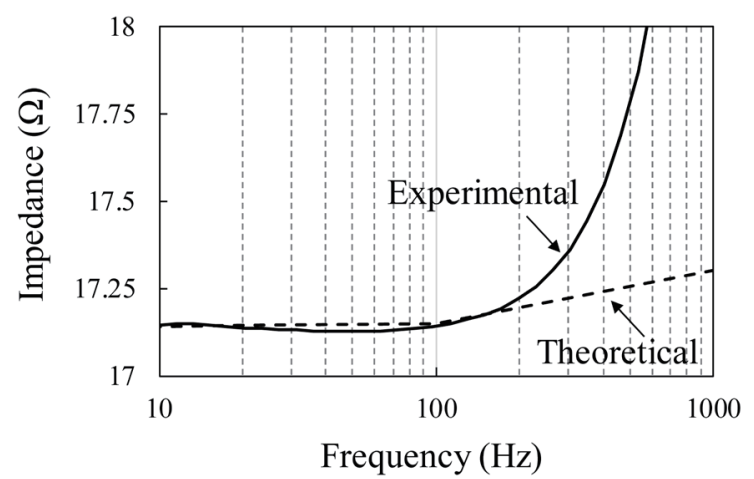

Fig. 9. Impedance-frequency characteristics of electromagnetic drive coil.

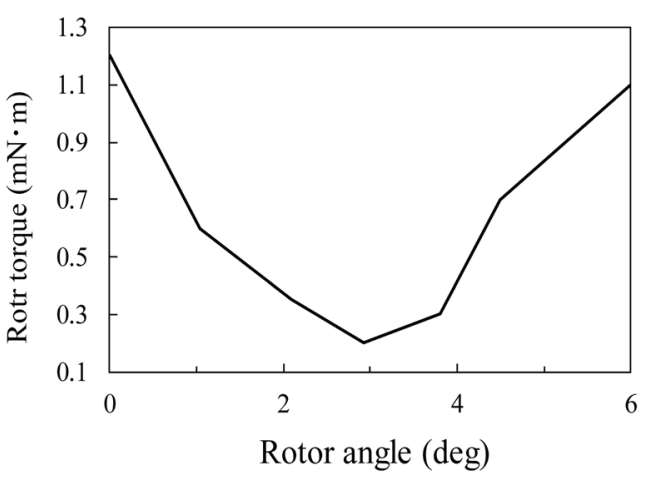

Fig. 10. Relationship of the torque of liquid feed rotor with rotation angle. 
liquid feed rotor increases the load that the rolling elements of the thrust bearing receive, resulting in energy loss due to the friction, and consequently, in the deterioration of the rotor torque. In order to prevent solution contamination, this micropump was used after cleaning all the components constituting the thrust bearing with acetone, and after removing oil such as a lubricant, the micropump was reassembled. The rolling elements were changed from the metal spheres to the nonmagnetic ceramic spheres, which are unaffected by the magnetic field, although the accuracy of the surface roughness decreases. Therefore, the load increase on the rolling elements can cause significant energy loss. However, because the use of a lubricant enables the smooth rotation of rolling elements and can be expected to improve the torque, it is useful to include lubrication in the future device design. In this case, it will be necessary to select the material considering the affinity between the magnetic fluid and the lubricant. This is because, when the affinity between the magnetic fluid and the lubricant is high, they easily mix with each other and the sealing performance is deteriorated.

\subsection{Results of liquid feeding performance evaluation}

When an $\mathrm{AC}$ of $0.62 \mathrm{~A}_{\mathrm{p}-\mathrm{p}}$ and $10 \mathrm{~Hz}$ was supplied to the electromagnetic drive coil, the liquid feed rotor did not rotate, but oscillated up and down like a seesaw with the center shaft as a fulcrum. It is considered that the attractive and repulsive forces acting between the electromagnetic drive coil and the neodymium magnet were strong, and the gap between the liquid feed rotor and the stator could not be kept constant, resulting in force deviation between the rotor and the stator; therefore, the rotor and stator vibrated up and down without rotating. Accordingly, when the current was gradually lowered and the electromagnetic force was weakened, the liquid feed rotor stably rotated at the current of $0.55 \mathrm{~A}_{\mathrm{p}-\mathrm{p}}$ and $10 \mathrm{~Hz}$. The rotational speed was gradually increased by raising the frequency. Up to 1150 rotations per minute, stable rotations were possible. Beyond 1200 rotations per minute, the rotation of the liquid feed rotor stopped as it became out of step. From these results, the upper limit of the rotational speed of the liquid feed rotor was evaluated to be 1000 rotations per minute in the liquid feeding experiments.

First, the discharge pressure of the micropump was evaluated. The discharge pressure was calculated from the change in the height of pure water in the waste beaker at the rotor rotational speeds of 200-1000 rotations per minute. Figure 11 shows the relationship of the discharge pressure with the rotation speed of the liquid feed rotor. The experimental result indicates that the discharge pressures were significantly reduced to about $5.6 \%$ at 1000 rotations per minute compared with the flow analysis result. Next, the flow rates at the rotor speeds of 500 and 1000 rotations per minute were calculated. The experimental results are shown in Table 1. The flow rates were 2 and $8 \mathrm{ml} / \mathrm{min}$, respectively. The flow rate at the rotor speed of 1000 rotations per minute was reduced to about $27 \%$ compared with the flow analysis result. The difference between the experimental result and the flow analysis result is considered to be caused by the following three factors. First, the weight of pure water in the micropump was not considered in the flow analysis; however, gravity acted on the actual pure water, resulting in the differences in the discharge pressure and flow velocity. Second, because the pipe resistance inside the 


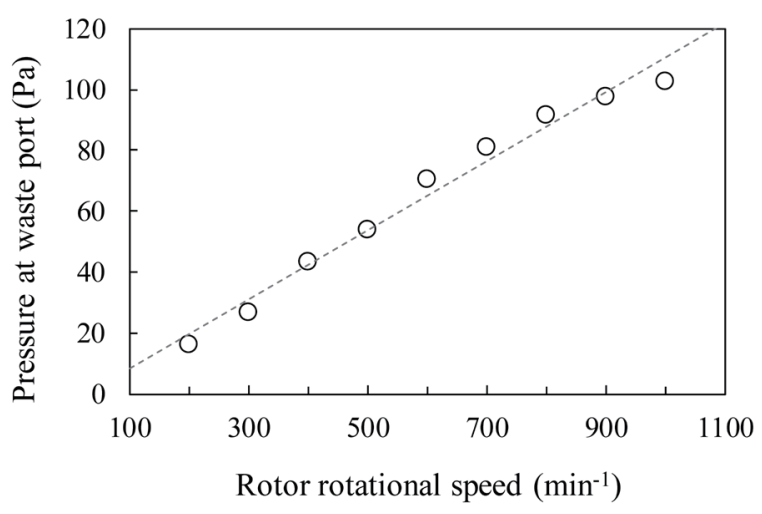

Table 1

Flow rate and rotor rotational speed.

\begin{tabular}{lrc}
\hline & \multicolumn{2}{c}{ Rotor rotational speed $\left(\mathrm{min}^{-1}\right)$} \\
\cline { 2 - 3 } & 500 & 1000 \\
\hline Flow rate $(\mathrm{ml} / \mathrm{min})$ & 2 & 8
\end{tabular}

Fig. 11. Relationship of the discharge pressure with respect to the rotor rotational speed.

tube through which the pure water was fed and the waste liquid was discharged was not taken into account in the flow analysis of the discharge pressure at the waste liquid port, differences in discharge pressure and the flow velocity occurred. Third, because of the load increase in the thrust bearing owing to electromagnetic attractive force, the frictional force increased, and therefore, the rotor torque decreased markedly, resulting in a difference between the discharge pressure and the flow velocity. To resolve these issues, improvement of discharge pressure and flow velocity can be expected upon expanding the blade area or changing the streamline shape, or narrowing the gap between the blade and the inner wall of the micropump. Furthermore, the reduction in friction force by using a lubricant for the thrust bearing is also effective. However, the flow rates of several $\mathrm{ml} / \mathrm{min}$ obtained in the experiment is sufficient for use in biomedical analysis. Moreover, the pump performance satisfies the specifications.

Subsequently, with pure water filled into the pump interior, feed tube, and feed waste beaker, it was evaluated whether or not the magnetic fluid seal was peeled off at the rotor rotation speeds of 200-1000 rotations per minute. As a result, we found that the pure water did not enter into the thrust bearing at 500 rotations per minute or less. At 600 rotations per minute and above, the pure water entered the thrust bearing, causing water droplets to adhere to the rolling elements. Figure 12 shows the evaluation results of whether or not pure water had entered into the thrust bearing with the magnetic fluid seal, at the rotor speeds of 500 and 600 rotations per minute. The magnetic fluid was neither peeled off nor attached to the electromagnetic drive magnet embedded in the liquid feed rotor; moreover, no mixing of the magnetic fluid in the waste pure water was observed up to 1000 rotations per minute. From the above results, it was found that the magnetic fluid seal for thrust bearings is effective at the rotor speed of 500 rotations per minute or less, and that liquid feeding adequate for use in the biomedical analyses can be realized. In the future, improvement of the magnetic fluid seal for the thrust bearing can be expected by enlarging the area of the ring neodymium magnets, which retain the magnetic fluid, and narrowing the gap between the ring neodymium magnets. 


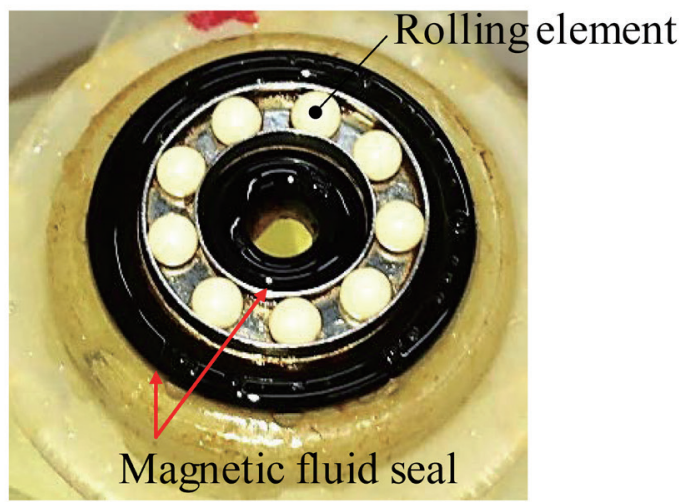

(a)

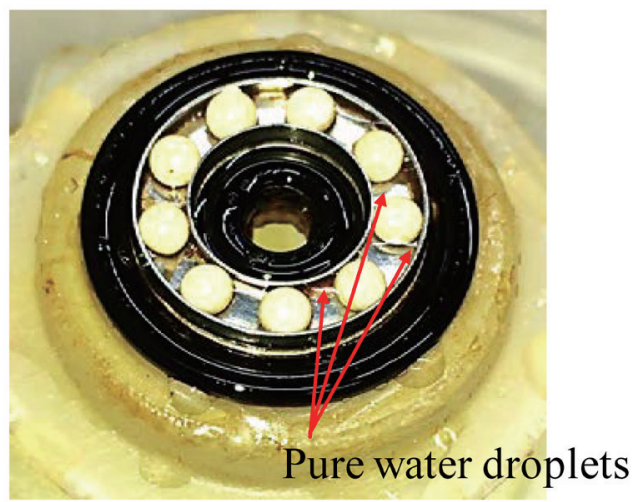

(b)

Fig. 12. (Color online) Evaluation result of whether pure water intrudes into the thrust bearing with magnetic fluid seal. Bearings inside after (a) 500 rotations per minute (without intrusion) and (b) 600 rotations per minute (with intrusion).

Because this study is a fundamental investigation of a novel micropump contributing to the miniaturization of medical analysis sensor systems, commercially available thrust bearings and neodymium magnets were used. The thrust bearings and neodymium magnets that composed the micropump were relatively smaller than other commercially available products. However, even if we wanted to reduce the diameter of the inlet/outlet and the magnetic fluid seal structure, the reduction was restricted by the dimensions of the bearing and magnet. It was found in this study that the magnetic-fluid-sealed thrust bearing is useful; therefore, in the future, a more compact micropump could be realized by manufacturing and using smaller and dedicated thrust bearings and neodymium magnets.

As the pump housing is made smaller, the electromagnetic drive coils must be made smaller. When the mutual induction between the coils is ignored, the electromagnetic force of the coil generated momentarily is theoretically proportional to the product of the current and the number of turns. Therefore, in this study, a current close to the allowable current was applied to obtain a high electromagnetic force. As a result, the coil generated heat at a high temperature $\left(50-60{ }^{\circ} \mathrm{C}\right)$, which caused the heating of even the solution inside the micropump. Although the temperature characteristics of the AT-cut quartz oscillator can be ignored near room temperature, the extent to which the temperature of the solution changed owing to the heat generated by the coil requires a temperature control system. The developed micropump needs to rotate the liquid feed rotor at about 500 revolutions per minute or more in order to obtain a liquid feeding amount of several microliters. If the liquid feed rotor rotates at such a high speed, the analyte (e.g., protein) may be physically damaged inside the pump chamber. From these results, it is necessary to pay attention to the following points when integrating the micropump with the PDMS-QCM biosensor chip. The solution should be fed from the sensor chip to the pump, not from the pump to the sensor chip. In this way, by using it as a suction pump, it is possible to avoid changes in solution temperature owing to the heat generated by the coil and damage to the analyte owing to the rotational force of the rotor. 


\section{Conclusion}

In this study, we proposed the electromagnetically driven micropump with a built-in magnetic-fluid-sealed thrust bearing that can be integrated with the PDMS-QCM biosensor chip, and showed the usefulness of the device through design, fabrication, and evaluation. The structural design of the micropump was carried out on the basis of the results of electromagnetic analysis and flow analysis, and the component parts were produced by the MJ method. Through the evaluation of the impedance characteristics of the electromagnetic drive coil, it was found that resonance or dip does not occur at low frequencies $(100 \mathrm{~Hz}$ or less) and that the effect of the parasitic capacitance between the electromagnetic drive coils was negligible. In the experiments using pure water, the liquid could be stably fed at the rotor speed of 1000 rotations per minute or less. At this time, it was found that the rotor speed was in direct proportion to the discharge pressure. At the rotor speed of 500 rotations per minute or less, the magnetic fluid seal functioned well, and the pure water did not enter into the thrust bearing. This result proved that the magnetic fluid sealing mechanism is applicable to a micropump using a thrust bearing. However, it is desirable to apply the developed micropump as a suction pump because the heat generated in the drive coil is high and the rotor speed is high. The integration of the micropump developed in this study and the PDMS-QCM sensor chip will enable further miniaturization of medical analytical sensor systems. As a result, such sensor systems will become more widely used, and the burden on patients during diagnosis and examination can be greatly reduced. The developed micropump technology will open up a new door for small medical analysis systems in the future.

\section{Acknowledgments}

This study is supported by the Suzuki Foundation, and in part by the Japan Society for the Promotion of Science (JSPS) KAKENHI Grant Number 17K01420.

\section{References}

1 F. Neumann, N. Madaboosi, I. Hernández-Neuta, J. Salas, A. Ahlford, V. Mecea, and M. Nilsson: Sens. Actuators, B 273 (2018) 742.

2 B. Zhou, Y. Hao, D. Long, and P. Yang: Biosens. Bioelectron. 111 (2018) 90.

3 H. J. Park and S. S. Lee: Sens. Actuators, B 258 (2018) 836.

4 C. K. O'Sullivan and G. G. Guilbault: Biosens. Bioelectron. 14 (1999) 663.

5 W. Chen, F. Deng, M. Xu, J. Wang, Z. Wei, and Y. Wang: Sens. Actuators, B 273 (2018) 498.

6 J. Krim: Nano Today 2 (2007) 38.

7 P. Sun, M. Wang, L. Liu, L. Jiao, W. Du, F. Xia, M. Liu, W. Kong, L. Dong, and M. Yun: Appl. Surf. Sci. 475 (2019) 342 .

8 J. Homola, S. S. Yee, and G. Gauglitz: Sens. Actuators, B 54 (1999) 3.

9 B. Meshginqalam and J. Barvestani: Opt. Mater. 86 (2018) 119.

10 A. S. Kushwaha, A. Kumar, R. Kumar, and S. K. Srivastava: Photonics Nanostruct. Fundam. Appl. 31 (2018) 99.

11 B. A. Adducci, H. A. Gruszewski, P. A. Khatibi, and D. G. Schmale: Biosens. Bioelectron. 78 (2016) 160.

12 S. S. Zhao, N. Bukar, J. L. Toulouse, D. Pelechacz, R. Robitaille, J. N. Pelletier, and J. Masson: Biosens. Bioelectron. 64 (2015) 664.

13 F. Kato, H. Noguchi, Y. Kodaka, N. Oshida, and H. Ogi: Jpn. J. Appl. Phys. 57 (2018) 07LD14-1-07LD14-1-9. 
14 F. Kato, H. Noguchi, Y. Kodaka, N. Chiku, H. Shibata, F. Abe, and H. Ogi: Proc. Transducers 2017 (2017) 1664-1667.

15 Y. Saigusa: Advanced Piezoelectric Materials, Second Edition, Science and Technology (Woodhead Publishing, Cambridge, 2017) p. 197.

16 S. Lee, Y. Kuan, and M. Sung: J. Power Sources 238 (2013) 290.

17 J. G. Smits: Sens. Actuators, A 21 (1990) 203.

18 X. Zhu, Y. Deng, P. Li, D. Yuan, and J. Ma: Microchem. J. 145 (2019) 1135.

19 M. S. V. Appaji, G. Shivakanth Reddy, S. Arunkumar, and M. Venkatesan: Procedia Mater. Sci. 5 (2014) 1791.

20 J. Xiang, Z. Cai, Y. Zhang, and W. Wang: Sens. Actuators, A 251 (2016) 20.

21 C. Koch, V. Remcho, and J. Ingle: Sens. Actuators, B 135 (2009) 664.

22 C. N. Pai and T. Shinshi: Med. Eng. Phys. 33 (2011) 906.

23 H. Onuma, M. Murakami, and T. Masuzawa: ASAIO J. 51 (2005) 50.

24 B. Pečar, M. Možek, and D. Vrtačnik: Inform. Midem. 47 (2017) 147.

25 T. Yamamoto: Method for Bonding Synthetic Resin (2013) WO2013042377A1. 